\title{
AN INNOVATIVE APPROACH TOWARD GAIT FEATURE DETECTION IN CHILDREN WITH CP
}

Nieuwenhuys Angela ${ }^{\mathrm{a}}$, Meyer Christophe ${ }^{\mathrm{a}}$, Monari Davide ${ }^{\mathrm{b}}$, De Laet Tinne ${ }^{\mathrm{b}}$, Van Gestel Leen ${ }^{\mathrm{a}}$, Molenaers Guy $^{\mathrm{c}, \mathrm{d}}$, Desloovere Kaat ${ }^{\mathrm{a}, \mathrm{d}}$

${ }^{\mathrm{a}}$ KU Leuven, Department of Rehabilitation Sciences, ${ }^{\mathrm{b}}$ KU Leuven, Department of Mechanical Engineering,

${ }^{\mathrm{c}}$ University Hospital Leuven, Department of Pediatric Orthopedics, ${ }^{\mathrm{d}}$ University Hospital Leuven, Clinical motion analysis laboratory, CERM. [Leuven, Belgium]

\section{INTRODUCTION and AIM}

Classifying gait patterns in children with cerebral palsy $(\mathrm{CP})$ and reducing the vast amount of data from gait analysis into a set of clinically relevant gait features remains a challenge [1]. A priori selection of gait features, often based on clinical expert knowledge, can result in either incomplete or redundant data [2]. The goal is to quantify differences in lower limb kinematics between $\mathrm{CP}$ children and typically developing controls using principal component analysis (PCA). Secondly, for features that differ between CP and controls, a discriminant analysis (DA) will be applied to determine which features discriminate best between both groups.

\section{PATIENTS/MATERIALS and METHODS}

$100 \mathrm{CP}$ gait trials and 72 age-matched control trials were retrospectively selected from the database of University Hospital Leuven. Children with spastic diplegia or hemiplegia (GMFCS level I-III), aged 5 to 12 years, without previous lower limb surgery and/or lower limb botulinum toxin treatment within 6 months prior to gait analysis were included. Pelvis, hip, knee and ankle joint kinematics were calculated from 3D gait analysis. PCA was applied to continuous waveform data using custom made MATLAB@ software (Mathworks, USA). The number of principal components (PCs) explaining $90 \%$ of the variability were retained. PC scores were calculated for each trial and analyzed for group differences using unpaired t-tests. Subsequently, DA was performed using the PCs that were significantly different between groups, following the approach of Deluzio et al. $[3,4]$. This method allows us to detect the three most discriminative features between groups.

\section{RESULTS}

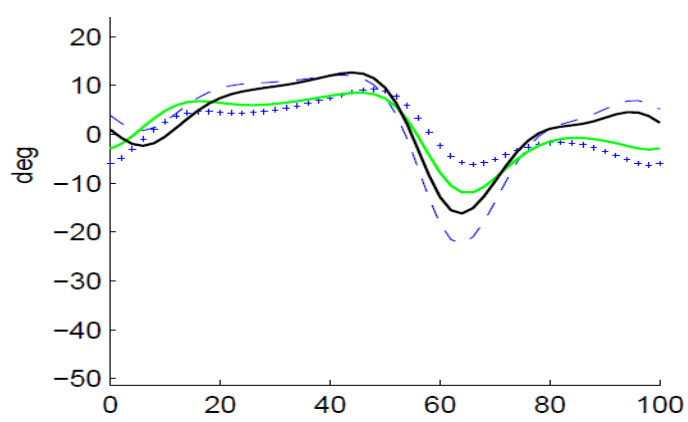

Figure 1. Mean ankle movement of $\mathrm{CP}$ (green) and controls (black) during one stride $(0-100 \%) .5^{\text {th }}(---)$ and $95^{\text {th }}(+++)$ percentile of PC2 scores of the ankle movement show that PC2 captures the peak plantar flexion during push-off.
Table 1. Description of principal components from lower limb joints in the sagittal plane. Mean (SD) PC scores and p-values are listed.

\begin{tabular}{|c|c|c|c|c|}
\hline PC & & $C P(n=100)$ & Control $(n=72)$ & p-value \\
\hline Pelvis & PC1 & $-20.24(37.94)$ & $28.12(30.84)$ & $<0.0001^{\star}$ \\
\hline \multirow[t]{3}{*}{ Hip } & PC1 & $-20.93(47.89)$ & $29.07(32.8)$ & $<0.0001^{*}$ \\
\hline & $\mathrm{PC} 2$ & $0.2(22.11)$ & $-0.27(15.99)$ & 0.8778 \\
\hline & PC3 & $-0.09(19.38)$ & $0.13(12.16)$ & 0.933 \\
\hline \multirow[t]{4}{*}{ Knee } & PC1 & $-18.87(49.13)$ & $26.21(37.5)$ & $<0.0001^{\star}$ \\
\hline & PC2 & $-14.1(32.47)$ & $19.59(23.51)$ & $<0.0001^{*}$ \\
\hline & PC3 & $-2.13(29.58)$ & $2.96(19.57)$ & 0.2046 \\
\hline & PC4 & $3.22(20.04)$ & $-4.47(13.07)$ & $0.005^{\star}$ \\
\hline \multirow[t]{4}{*}{ Ankle } & PC1 & 3.68 (54.9) & $-5.11(28.28)$ & 0.2153 \\
\hline & PC2 & $8.57(14.14)$ & $-11.9(13.13)$ & $0.0001^{\star}$ \\
\hline & PC3 & $-5.04(12.86)$ & $7.0(12.43)$ & $0.0001^{\star}$ \\
\hline & PC4 & $3.14(14.66)$ & $-4.36(10.37)$ & $0.0003^{\star}$ \\
\hline
\end{tabular}
t-test $(\alpha=.05)$

Only 4 PCs were not able to highlight differences between groups. The remaining 8 PCs were implemented in the discriminant analysis. The cross-validation results showed that $91.3 \%$ of all trials can be classified correctly and the coefficient of the PCs in the discriminant function were highest for the ankle-PC2 (0.71), the pelvis-PC1 (0.67) and the hip-PC (0.6).

\section{DISCUSSION and CONCLUSIONS}

Based on the PCA and linear DA, three PCs were defined as being the most important to discriminate between $\mathrm{CP}$ children and controls. PC2 in the ankle mainly represents plantar flexion motion during push-off, PC1 in the pelvis and in the hip both point towards an offset, i.e. a higher mean anterior tilt and higher hip flexion angle throughout a gait cycle for the $\mathrm{CP}$ group. This approach toward gait classification is relatively new in CP. Only one article has previously applied this method and found no significant differences in the sagittal plane between children classified as GMFCS level I and II [4]. In future research this method will be extended to larger sample sizes, across the three different planes and for various subgroups of children with $\mathrm{CP}$.

\section{REFERENCES}

[1] Dobson F et al. Gait Posture 25, 140-52 (2007). [2] Mantovani G et al. J Biomech 45, 2109-15 (2012).

[3] Deluzio KJ et al. Gait Posture 25, 86-93 (2007). [4] Kirkwood RN et al. ISRN pediatrics 2012, 1630-39 (2012). 